\title{
Argumentación de futuros profesores de química sobre la anorexia tratada como cuestión sociocientífica
}

\author{
Leonardo Fabio Martínez Pérez ${ }^{\mathbb{1}}$, Dora del Carmen Gallo Aldana ${ }^{(i)}$ \\ Universidad Pedagógica Nacional, Universidad Jorge Tadeo Lozano, Colombia
}

Autor de correspondencia: ${ }^{1}$ lemartinez@pedagogica.edu.co Recibido: 10 de octubre de 2020 Revisado: 14 noviembre de 2020 Aprobado: 26 de febrero de 2021 Publicado: 06 de junio de 2021

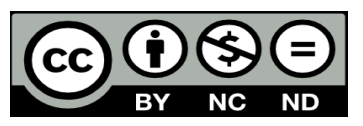

\section{Resumen}

En este artículo se analiza la argumentación de profesores de química en formación inicial a través del abordaje de una cuestión sociocientífica [CSC] sobre la anorexia asociada al metabolismo de aminoácidos. El trabajo siguió una metodología cualitativa microetnográfica. Se emplearon registros escritos y orales obtenidos durante la implementación de la secuencia basada en las implicaciones sociales de la anorexia. Para la fundamentación y análisis de los resultados, se utilizó el modelo de argumentación de Toulmin. El grado de argumentación alcanzado se determinó con los niveles argumentativos. Los resultados obtenidos indican que los niveles de argumentación predominantes en los profesores en formación son 0 y 1 , razón por la cual la argumentación debe incorporarse de forma permanente y transversal en los currículos de formación de los futuros profesores de química para que así puedan ser capaces de favorecer mejores procesos de argumentación. En cuanto a su posición sobre el empleo de una CSC controvertida como la anorexia, los profesores en formación evidencian una comprensión amplia de la enfermedad, situación que contribuye a desarrollar el pensamiento crítico.

Palabras clave: argumentación, enseñanza de la química, formación de profesores, innovación educacional 


\title{
Argumentation of future chemistry teachers on anorexia addressed as a socio-scientific issue
}

\begin{abstract}
This article analyzes the argumentation of chemistry trainee teachers through a socioscientific issue [SSI] on anorexia associated with the amino acid metabolism. The study followed a qualitative microethnographic methodology. We used written and oral records obtained during the implementation of the sequence based on the social implications of anorexia. Toulmin's argumentation model was used to support and analyze the results. The degree of argumentation achieved was determined using the argumentative levels. The results obtained indicate that the predominant levels of argumentation of the trainee teachers are 0 and 1 , which is why argumentation should be included in a permanent and transversal way in curricula for the training of future chemistry teachers so that they will be able to enhance the processes of argumentation. Regarding their position on the use of a controversial SSI such as anorexia, the trainee teachers show a broad understanding of the disease, which contributes to the improvement of critical thinking.
\end{abstract}

Keywords: argumentation, chemistry teaching, teacher training, educational innovation

\section{Argumentação de futuros professores de química sobre a anorexia abordada como uma questão sociocientífica}

\section{Resumo}

Este artigo analisa a argumentação dos licenciandos de química através de uma questão sociocientífica [QSC] sobre a anorexia associada ao metabolismo dos aminoácidos. O estudo seguiu uma metodologia qualitativa micro-etnográfica. Foram utilizados registros escritos e orais obtidos durante a implementação da sequência baseada nas implicações sociais da anorexia. $\mathrm{O}$ modelo de argumentação de Toulmin foi utilizado para apoiar e analisar os resultados. O grau de argumentação alcançado foi determinado utilizando os níveis argumentativos. Os resultados obtidos indicam que os níveis predominantes de argumentação dos licenciandos são 0 e 1, razão pela qual a argumentação deve ser incluída de forma permanente e transversal nos currículos de formação dos futuros professores de química para que eles possam reforçar os processos de argumentação. Quanto à sua posição sobre o uso de uma QSC controversa, como a anorexia, os licenciandos demonstram uma ampla compreensão da doença, o que contribui para a melhoria do pensamento crítico.

Palavras-chave: argumentação, ensino de química, formação de professores, inovação educacional 
La investigación en la enseñanza de las ciencias abarca el campo de la formación inicial y continuada de los profesores. Es de resaltar la importancia de diseñar e implementar propuestas innovadoras que consideren las necesidades formativas de los docentes y sus respectivos saberes para mejorar el aprendizaje de las ciencias.

Una propuesta que se destaca en el ámbito didáctico y que empieza a desarrollarse con gran ímpetu es la argumentación en cuestiones sociocientíficas [CSC], la cual permite el desarrollo del pensamiento crítico e investigativo de los jóvenes estudiantes que incursionan en el mundo de la enseñanza de la ciencia.

En la presente investigación se llevó a cabo un estudio cualitativo microetnográfico con un grupo de profesores de química en formación inicial [PQFI] de la Universidad Pedagógica Nacional [UPN] que cursaron el espacio académico de Sistemas Bioquímicos, con el objetivo de analizar el proceso de argumentación que realizan, a través del desarrollo de una secuencia de enseñanza sobre el metabolismo de aminoácidos, centrado en la CSC "Anorexia, un desorden de salud". En dicho trabajo se utilizó el modelo de Toulmin (2007), con el cual, a partir del dato "la anorexia es un trastorno de la conducta alimentaria" y la conclusión "por lo tanto, altera el metabolismo de aminoácidos", se analizó la argumentación de los PQFI. Para evaluarla, se utilizaron las propuestas de Driver et al. (2000) y de Moreno y Martínez (2009), quienes caracterizan diferentes niveles de argumentación establecidos por los estudiantes durante discusiones sobre cuestiones de ciencia.

La cuestión de la anorexia es de interés, debido a que no se presentan estudios acerca de argumentación en clases de Bioquímica sobre este tema que susciten eventuales controversias y temas de importancia social para los estudiantes. Ahora bien, es de anotar que el trabajo con CSC demanda la articulación de conocimientos científicos y sociales por parte del futuro profesor de química, así como el desarrollo de procesos argumentativos sobre problemáticas que afectan su entorno y que puedan favorecer a las comunidades con las que interactúa.

\section{Fundamentación teórica}

Este reporte de investigación se fundamenta en Toulmin (2007), quien propone un modelo lógico para entender los procesos argumentativos basado en una estructura coherente: elaboración de premisas a partir de datos, justificaciones y garantías de apoyo y una conclusión consistente.

En la figura 1 se presenta la estructura básica del argumento, en donde D son los datos y G las justificaciones que respaldan la construcción de la afirmación para concretar la conclusión, representada por C.

Esta estructura básica es ampliada por el mismo autor al hacer referencia a la incorporación de excepciones, garantías y calificativos que dan fortaleza al argumento para responder a refutaciones que se puedan realizar a la afirmación construida, tal como se ilustra en la figura 2. 
Figura 1

Estructura básica del argumento

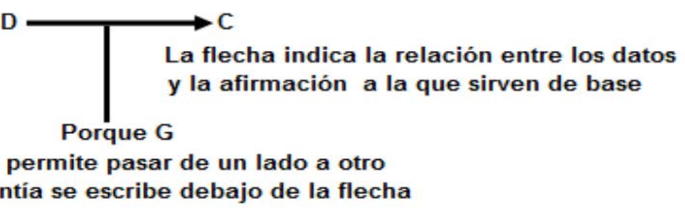

Nota. Tomada de Los usos de la argumentación, de S. Toulmin, 2007, p. 135.

Figura 2

Ampliación del esquema argumentativo

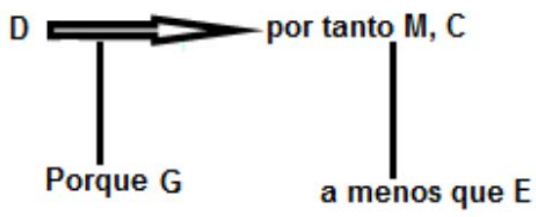

\section{M y E son diferentes de los datos y las garantías \\ $M=$ calificativo o matizador modal. Se escribe al lado de la conclusión que matiza (C) \\ $\mathrm{E}=$ concepciones de excepción o refutación. Se escribe debajo del calificativo}

Nota. Tomada de Los usos de la argumentación, de S. Toulmin, 2007, p. 138.

Para evaluar el proceso argumentativo en la enseñanza de las ciencias, es necesario hacer una contextualización del modelo de Toulmin a los procesos realizados en el aula, para lo cual retomamos investigaciones previas de Moreno y Martínez (2009), donde se retoma la propuesta de Driver y Newton (1997) para elaborar la tabla 1, que ilustra los distintos niveles de argumentación que podemos caracterizar cuando implementamos actividades didácticas para favorecer el aprendizaje de conceptos científicos. En esta tabla se ilustran las afirmaciones que se pueden caracterizar desde un nivel de argumentación básico 0 hasta un nivel avanzado 4. Con base en esta tipología de afirmaciones, se analizan los registros consolidados en esta investigación para establecer los niveles de argumentación de los PQFI.

Tabla 1

Niveles de argumentación adaptados del trabajo de Driver y Newton (1997)

\begin{tabular}{lcc}
\hline \multicolumn{1}{c}{ Afirmaciones abarcadas en el proceso argumentativo } & $\begin{array}{c}\text { Niveles de } \\
\text { argumentación }\end{array}$ & Símbolo \\
\hline Aislada sin justificación & 0 & AASJ \\
\hline Competitiva sin justificación & 0 & ACSJ \\
\hline Requerida sin justificación & 0 & ARSJ \\
\hline Sin justificación & 0 & ASJ \\
\hline Aislada con justificación & 1 & AICJ \\
\hline
\end{tabular}




\begin{tabular}{llc}
\hline Con justificación & 1 & ACJ \\
\hline Requerida con justificación & 1 & ARCJ \\
\hline Competitiva con justificación & 2 & ACCJ \\
\hline Con justificación y cualificador & 2 & ACJCU \\
\hline Competitiva con justificación y cuestionamiento & 2 & ACCJC \\
\hline Competitiva con cuestionamiento & 2 & ACCC \\
\hline Requerida competitiva con justificación & 2 & ARCCJ \\
\hline Competitiva con justificación y cualificadores & 3 & ACCJCU \\
\hline Requeridas compitiendo con justificación y cualificadores & 3 & ARCCJCU \\
\hline Competitivas con justificaciones respondiendo por refutación & 3 & ACCJRR \\
\hline $\begin{array}{l}\text { Requeridas compitiendo con justificación respondiendo por } \\
\text { refutación }\end{array}$ & 3 & ARCCJRR \\
\hline Juzgamiento integrando diferentes argumentos & 4 & JIDA \\
\hline
\end{tabular}

Nota. Adaptada de "Argumentación en estudiantes de educación media y habilidad del profesor para su desarrollo: una discusión en el aula sobre implicaciones sociales y ambientales de la producción de etanol”, de D. Moreno y L. Martínez, 2009, Nodos y Nudos p. 39.

\section{La anorexia como CSC}

El abordaje de CSC en la enseñanza de las ciencias, y en particular en la enseñanza de la química, puede contribuir a mejorar la práctica del profesor, ya que posibilita desarrollar procesos argumentativos en los estudiantes, promueve el pensamiento crítico, favorece la toma de decisiones y posibilita el establecimiento de relaciones entre la ciencia, la tecnología, la sociedad y el ambiente (Martínez, 2012).

Las CSC son asuntos controvertidos en la sociedad y tienen una base científica de actualidad. Con frecuencia se divulgan en medios de comunicación. Tienen efectos locales y/o globales. Implican, en la mayoría de casos, aspectos éticos y morales. Pueden comprender discusiones ambientales y generalmente, cuando son abordadas en clases de ciencias, conllevan el desarrollo de racionamientos informales y procesos de argumentación.

En Martínez (2014) se analiza cada una de estas características de las CSC en el caso de las controversias que abarca la producción de biocombustible a partir de etanol. Según esta misma perspectiva, en la tabla 2 presentamos las características de la anorexia tratada como una CSC.

Tabla 2

Características de la CSC

Características de las CSC

Se basan en la ciencia, por lo general en áreas que están en las fronteras del conocimiento científico.
Controversias asociadas a la anorexia

Si bien es cierto que la anorexia es considerada una enfermedad asociada a un trastorno alimentario de origen psicológico emocional, el diagnóstico implica un abordaje multidisciplinar que abarca un componente científico que aún tiene vacíos importantes. Por ejemplo, existen estudios hipotéticos que podrían hacer alusión a una causa genética. En este sentido, las bases científicas de la enfermedad son controvertidas e implican investigaciones contemporáneas en desarrollo. 
Por lo general, las divulgan los medios de comunicación masiva, al destacar aspectos relacionados con sus intereses.

Enfrentan problemas locales y globales.

Abarcan la formación de opiniones y la realización de elecciones en los niveles personal y social.

Enfrentan información incompleta, ya se trate de evidencias científicas incompletas o confrontantes, o de vacíos en los registros.

- Abarcan análisis de costo y beneficio en los cuales los riesgos interactúan con los valores.

- Pueden requerir algún entendimiento de probabilidad y riesgo.

Pueden abarcar consideraciones sobre sustentabilidad.
Teniendo en cuenta que la anorexia es una enfermedad asociada a adicciones psicológicas por seguir determinados estereotipos sociales, frecuentemente en los medios de comunicación se divulgan noticias sobre el fallecimiento de reconocidas personalidades por causa de la enfermedad, entre otros temas asociados.

Se encuentran diversos casos de estudio sobre las implicaciones sociales de la anorexia. Se destacan temas asociados a la prevención de la enfermedad en distintos contextos sociales y culturales del ámbito local y global.

La complejidad de la enfermedad abarca gran cantidad de opiniones personales que determinan comportamientos concretos de rechazo a una alimentación normal.

Se encuentran diversos casos de estudio sobre las implicaciones sociales de la anorexia. Se destacan temas asociados a la prevención de la enfermedad en distintos contextos sociales y culturales del ámbito local y global.

La complejidad de la enfermedad abarca gran cantidad de opiniones personales que determinan comportamientos concretos de rechazo a una alimentación normal.

Las críticas ambientales sobre la sociedad de consumo son importantes para buscar escenarios de valorización y autoestima personal.

La pérdida de autonomía y autoestima corporal abarcan discusiones sobre principios éticos tales como el cuidado de la vida, pues la enfermedad en los estados más críticos termina en la muerte de quien la padece.

\section{Metodología}

En la investigación se empleó la microetnografía, que consiste en focalizar el trabajo de campo a través de la observación e interpretación del fenómeno en una sola institución social, o en una o varias situaciones sociales. La investigación implica un trabajo restringido que amerita poco tiempo y que se puede desarrollar por un solo investigador (Murillo \& Martínez, 2010). Este tipo de etnografía también se denomina de la comunicación, porque se centra en el análisis de los patrones con los que interactúan los miembros de una comunidad, con énfasis en las cuestiones sociolingüísticas (Álvarez, 2008).

Desde la perspectiva de Zubieta (1982), la microetnografía, además de centrarse en la institución educativa, se concreta en la interacción profesor-estudiante en el aula de clase. El principal aporte de este tipo de estudios ha sido enriquecer el análisis de hechos educativos. De otro lado, ha tenido acogida en los sistemas educativos y por parte de los docentes, ya que ofrece una orientación valiosa para ser más eficiente su labor.

Según Moreira (2002), este tipo de investigación se ocupa de mirar repetidas veces y analizar detalladamente registros audiovisuales de interacciones humanas en escenas clave, situaciones también esenciales de interacciones sociales, acompañadas de observaciones participativas. Es comunicativa porque enfoca a los sujetos individuales y sus discursos en ciertos escenarios. En esta metodología se trata de analizar e interpretar la información verbal y no verbal proveniente de un trabajo de campo, cuyos datos consisten en experiencias textuales de los protagonistas del fenómeno o de la observación realizada en el ambiente natural para comprender lo que hacen, dicen y piensan sus actores, además de cómo 
interpretan su mundo y lo que en él sucede.

Con base en los autores citados, se consideró pertinente realizar la investigación con la metodología cualitativa de microetnografía, porque se ajusta en buena medida a los parámetros de esta clase de investigación, no solo en la descripción del contexto que se va a trabajar, sino porque permite una análisis discursivo de las interacciones que se establecieron con futuros profesores de química durante la implementación de la secuencia de enseñanza referida al metabolismo de aminoácidos, a partir de discusiones sobre aspectos sociales y de salud contenidos en la cuestión de la anorexia. La investigación se desarrolló de acuerdo con las fases que se describen a continuación.

\section{Fase I. Diseño de la secuencia de enseñanza}

Las secuencias de enseñanza que tienen mayor éxito son aquellas en las que el profesor se involucra con el pensamiento de los estudiantes en el tema propuesto y elabora una secuencia de enseñanza que logra interesarlos y satisfacerlos intelectualmente (Zenteno \& Garritz, 2010). Leach y Scott (2002) especifican que, antes de desarrollar la secuencia de enseñanza, se deben considerar los siguientes aspectos: (a) identificar el conocimiento que se va a enseñar; (b) tener en cuenta cómo se aborda en el lenguaje social cotidiano de los estudiantes el tema que se quiere tratar; y (c) identificar la demanda de aprendizaje.

La secuencia de enseñanza propuesta se basa en la argumentación de una CSC. Se elaboró teniendo en cuenta que va dirigida al espacio académico de Sistemas Bioquímicos del programa de Licenciatura en Química de la UPN. Allí se busca aportar a la formación inicial de los profesores de química en articulación con la visión del Departamento de Química de la UPN, orientado a comprender los procesos educativos sociales y culturales del país en el contexto del desarrollo de profesionales competentes en el área de la educación en química, y en particular de la bioquímica.

Este espacio académico se justifica porque existe un aumento de las investigaciones científicas asociadas a los mecanismos químicos que rigen la vida y su importancia social, tecnológica y ambiental, razón por la cual es pertinente y relevante que el futuro profesor de química argumente y escriba de acuerdo con los conocimientos generados en el área de la bioquímica. Esta asignatura - dirigida en su momento por el coautor de esta investigacióncomprende los siguientes núcleos de trabajo:

- Primer núcleo: las enzimas y su importancia teórica y experimental en el surgimiento de la bioquímica como campo de investigación.

- Segundo núcleo: metabolismo de glúcidos, lípidos y aminoácidos.

Para su desarrollo, se estructuraron seminarios en donde se valoró la participación crítica de los estudiantes, su capacidad de argumentación y su producción escrita. Ellos debían estudiar con anticipación el material pertinente. De este espacio académico, se escogió el metabolismo de aminoácidos, con el que se elaboró la secuencia de enseñanza que se analizó en esta investigación. Se tuvo en cuenta que el tema se relacionaba directamente con la cuestión de la anorexia, de interés por sus implicaciones sociales y en la salud humana.

La secuencia contiene los siguientes aspectos:

- Contextualización. Acerca de una enfermedad asociada con el metabolismo de los 
aminoácidos.

- Apuntes históricos. Muestra cómo este tipo de trastornos se ha generado por diversas razones que incluyen aspectos psicológicos, sociales y culturales.

- Puntos de reflexión. Hay preguntas, videos y noticias relacionadas con la anorexia. Esto permite llevar al PQFI a reflexionar sobre la CSC de la anorexia.

- Preguntas orientadoras que introducen al tema de los aminoácidos y su metabolismo. Cada una ofrece ítems para consultar, lo cual tiene como objetivo que el PQFI profundice sobre el tema para que estructure argumentos para participar en las discusiones en clase.

- Actividad experimental. Se realiza un análisis de orina en la que el PQFI podrá encontrar algunos de los metabolitos producidos en el metabolismo de aminoácidos en el contexto de su estudio sobre la anorexia.

\section{Fase II. Implementación de la secuencia y análisis}

La implementación de la secuencia se desarrolló en tres sesiones. En la primera, se presentó un video de anorexia. Luego se realizó un debate con el objetivo de identificar la forma de argumentar de los PQFI. Las sustentaciones se grabaron y los PQFI entregaron un documento con el mismo procedimiento.

En la segunda sesión se trabajó con la secuencia de acuerdo con los siguientes aspectos:

- un caso real: Las voces de la anorexia (Suárez, 2009).

- una parte histórica.

- temas de consulta alrededor del metabolismo de aminoácidos.

- tema de laboratorio.

En la tercera sesión, se realizó una práctica sobre el análisis cualitativo de aspectos fisicoquímicos de la orina, especialmente de cuerpos cetónicos, algunos iones y proteínas, actividad que permitió la comprensión del metabolismo de los aminoácidos en el marco del estudio de la anorexia.

En la última sesión, se llevó a cabo un segundo debate sobre Las voces de la anorexia (Suárez, 2009). Se les pidieron a los PQFI razones justificadas en los conocimientos bioquímicos, sobre la alteración del metabolismo de aminoácidos que sufre la paciente. Así mismo, podían plantear posibles tratamientos para mejorar la salud de estos enfermos.

Para la recolección de la información, se utilizaron el cuestionario (anexo 1), que permitió caracterizar a los PQFI, y las grabaciones de audio, para llevar un registro sobre las argumentaciones de los PQFI durante las actividades programadas. Finalmente, se desarrolló una entrevista semiestructurada para consolidar otros registros sobre la implementación de la secuencia y sobre los procesos de argumentación de los PQFI.

\section{Resultados}

\section{Caracterización de los participantes de la investigación}

El grupo participante estuvo constituido por 24 PQFI de la Facultad de Ciencia y 
Tecnología de la UPN, inscritos en la asignatura de Sistemas Bioquímicos, de la Licenciatura en Química. Se caracterizó el grupo mediante un cuestionario para obtener información sobre los siguientes puntos:

1. Edad: oscilaron entre 20 a 30 años.

2. Semestre: 22 cursaban octavo y 2 noveno.

3. Asignaturas que incluían argumentación y/o CSC: materias como Pedagogía y Didáctica I, II y III involucran argumentación y CSC, mientras que asignaturas como Sistemas Inorgánicos, Teorías Químicas, Métodos de Análisis, Sistemas Bioquímicos, entre otras, no toman en cuenta esta perspectiva como estrategia innovadora de la enseñanza.

4. Implementación de CSC y/o argumentación en la práctica docente: solo la mitad de los PQFI había efectuado su práctica docente. Dentro de las actividades que realizaron, había algunas propuestas que incluían argumentación mediante propuestas escritas y sustentadas. De igual forma, los temas que más tenían relación con CSC fueron aquellos que incluían cuestiones ambientales.

5. Manejo de los conocimientos adquiridos en los programas de Química, Pedagogía y Didáctica para la interpretación de las reacciones que ocurren en el aminoácido triptófano para convertirse en serotonina. Se empleó un caso ficticio sobre un paciente con síntomas relacionados con la salud, con falta de sueño y de apetito, entre otros, los cuales podían estar relacionados con los bajos niveles de serotonina. Para el análisis se utilizaron tres aspectos:

- Forma iónica del aminoácido triptófano a pH fisiológico: se encontró que algunos PQFI relacionaron el concepto de ionización de este tipo de biomoléculas a este $\mathrm{pH}$, mientras que otros utilizaron conceptos químicos no relacionados.

- Cambios químicos que tiene el triptófano hasta convertirse en serotonina: contrario a lo anterior, se encontró que la mitad de los PQFI reconocían reacciones de hidroxilación y descarboxilación, pero solamente una minoría podía interpretar en el mismo proceso ambas reacciones.

- Forma iónica del aminoácido triptófano a pH fisiológico: se encontró que algunos PQFI relacionaron el concepto de ionización de este tipo de biomoléculas a este $\mathrm{pH}$, mientras que otros utilizaron conceptos químicos no relacionados.

6. Argumentos a favor o en contra sobre el dictamen médico. 11 PQFI apoyan el diagnóstico sin tener argumentos que validen este juicio. De otro lado, los que no lo apoyan dicen no conocer este tipo de aminoácido o simplemente lo catalogan como no esencial, porque se puede formar en el organismo, lo cual evidencia la falta de conocimiento acerca de la clasificación de los aminoácidos como esenciales o no esenciales.

7. La forma en que argumentan con el uso de conceptos bioquímicos acerca de una cuestión social. Se propuso como tema de argumentación la muerte de una niña causada por hambre. Se encontraron argumentos generales basados en conceptos tales como tipo de enlace entre aminoácidos, función de las proteínas, aminoácidos esenciales y no 
esenciales, así como los que relacionan los conceptos bioquímicos de estas biomoléculas con la formación de acetilCoA, energía, formación de tejido como el colágeno, entre otros.

Estos resultados indican la importancia de la participación de los PQFI en el análisis de contenidos curriculares que les permitan tener una formación más apropiada de la química (Sangiogo et al., 2011). A la vez, pueden aproximarse a la investigación científica y elaborar preguntas adecuadas relacionadas al proceso de enseñanza aprendizaje, lo cual se verá reflejado en futuras prácticas docentes, de modo que consideren los conceptos escolares como un proceso complejo y permanente.

\section{Caracterización de niveles de argumentación}

De acuerdo con el objetivo general de la investigación, orientado hacia el análisis de los procesos de argumentación desarrollados por PQFI, y en particular al nivel alcanzado durante la implementación de la secuencia didáctica definida en la metodología, se utilizó la teoría de análisis de argumentación descrita por Liakopoulos (2002), quien se fundamenta en la teoría de la argumentación de Toulmin (2007) y, conforme a esta, caracteriza detalladamente las partes de un argumento en cualquier registro escrito en proposición central, datos, garantías, apoyos y refutación.

En la representación esquemática de Toulmin, el dato a estudiar fue "la anorexia es un trastorno de la conducta alimentaria" y la conclusión "por lo tanto, altera el metabolismo de los aminoácidos". Las garantías - que validan el dato para llegar a la conclusiónestán conformadas por los conceptos bioquímicos relacionados con el metabolismo de los aminoácidos, como son el origen y la utilización de estos biocompuestos.

Para interpretar el argumento bioquímico del metabolismo de los aminoácidos y el relacionado con la parte social de la enfermedad — expuesto por los PQFI-, se definieron dos unidades de análisis, una escrita y una oral, las cuales se refieren explícitamente a los puntos de vista de los autores del debate (Liakopolus, 2002):

- Escrito y oral: caracterización de los argumentos bioquímicos, su relación con el metabolismo de aminoácidos y nivel argumentativo.

- Oral: caracterización de los argumentos relacionados con la toma de posición en torno a una cuestión social: la anorexia.

Para determinar el nivel argumentativo, se tomó como referencia el modelo de Driver et al. (2000), adaptado por Moreno y Martínez (2009). A partir de este, se elaboró la tabla 3 para que tuviera específicamente en cuenta la pertinencia de los argumentos bioquímicos relacionados con el metabolismo de los aminoácidos.

De acuerdo con lo anterior, en textos grupales de los PQFI se encontraron proposiciones que involucraban razonamientos bioquímicos centrados en particularidades de los aminoácidos, pero no tuvieron en cuenta aspectos como el tipo de reacciones que se llevan a cabo en la obtención de biopolímeros, como las proteínas, ni en la producción de anticuerpos, entre otros. Dado que las anteriores afirmaciones no están directamente relacionadas con el metabolismo de los aminoácidos, pero se justifican bioquímicamente, se encuentran en un nivel 1 de argumentación. 
Tabla 3

Niveles de argumentación

\begin{tabular}{cl}
\hline $\begin{array}{c}\text { Nivel de } \\
\text { argumentación }\end{array}$ & Característica de las proposiciones bioquímicas realizadas durante el proceso \\
\hline 0 & $\begin{array}{l}\text { Comprende afirmaciones aisladas sobre aspectos de los procesos metabólicos no } \\
\text { justificadas. }\end{array}$ \\
\hline 0 & Comprende afirmaciones requeridas sobre el tema sin justificación. \\
\hline 1 & Comprende afirmaciones aisladas sobre el tema que tienen una justificación. \\
\hline 1 & $\begin{array}{l}\text { Comprende afirmaciones que son requeridas por el dato y alcanzan a tener una } \\
\text { justificación científica. }\end{array}$ \\
\hline 2 & Comprende afirmaciones competitivas y que presentan una justificación científica. \\
\hline 2 & Comprende afirmaciones competitivas con cuestionamiento. \\
\hline
\end{tabular}

Nota. Tomada de Argumentación en profesores de Química en formación inicial a partir de una secuencia de enseñanza sobre metabolismo de aminoácidos, de Gallo, 2013, p. 71.

Los que presentan afirmaciones con justificaciones - al describir los eventos que ocurren cuando un anoréxico se enfrenta a un ayuno prolongado- incluyen algunos conceptos que apoyan científicamente la necesidad de los aminoácidos, como el balance de nitrógeno, indispensable en el organismo para comprender la falta de proteogénesis en las personas anoréxicas, y la cirrosis como consecuencia de la falta de trabajo hepático. También presentan afirmaciones confusas, pero justificadas. Al igual que en el anterior conjunto de proposiciones, no llegan a la conclusión propuesta, lo cual los ubica en un nivel argumentativo 1 .

En cuanto a los textos individuales, había afirmaciones en las que los aspectos bioquímicos estaban dispersos y no existía una comprensión adecuada acerca de la obtención y el metabolismo de aminoácidos provenientes de las proteínas endógenas. Por lo tanto, las afirmaciones fueron requeridas sin justificación, lo que corresponde a un nivel argumentativo 0 .

Había afirmaciones centradas en el uso de los aminoácidos en la formación de proteínas, las cuales tienen diferentes funciones - de contracción muscular, de soporte estructural, de obtención de energía, hormonal, de transporte-. Estas proposiciones, aunque son requeridas con justificación, presentan un escaso lenguaje científico, lo que permitió clasificarlas en un nivel argumentativo 1.

Hay quienes presentan algunos términos científicos relacionados con el metabolismo oxidativo y evidencian tener claridad sobre la obtención de energía a partir de biomoléculas - carbohidratos, lípidos y proteínas-. Asimismo, usan conceptos relacionados con la obtención de energía a partir de biomoléculas - carbohidratos, lípidos y proteínas que se pueden obtener a partir de las reservas de glucógeno, grasa y tejidos corporales-. Además, despliegan garantías y apoyos que expresan aspectos del metabolismo oxidativo. Sin embargo, no hay argumentos amplios sobre el metabolismo de aminoácidos. Por lo tanto, también son afirmaciones requeridas con justificación para un nivel argumentativo 1.

Por su parte, hay textos que presentan un vocabulario relacionado con el metabolismo de los aminoácidos - transaminación, transformación del esqueleto carbonado en glucosa y cuerpos cetónicos, triacilgliceroles, gluconeogénesis y glucogenólisis-. Estos términos permiten expresar argumentos con justificaciones necesarias para un debate relacionado con 
el tema. Las afirmaciones y apoyos permiten llegar con cierta profundidad a la conclusión planteada. Por lo tanto, se pueden ubicar en un nivel argumentativo 2.

De otro lado, las discusiones argumentadas mostraron la falta de claridad sobre el orden en que actúan las vías metabólicas y un conocimiento superficial de la glucólisis, de la lipólisis y de la proteólisis. Esto implica que el participante de una discusión oral debe ordenar los argumentos que va a emplear, con el objetivo de trabajarlos y sostenerlos convincentemente (Marafioti, 2006). Desde este punto de vista, son proposiciones aisladas sobre el tema que tienen una justificación, lo cual corresponde a un nivel argumentativo 1.

Hubo afirmaciones que se basaron únicamente en el orden de la degradación de moléculas y dejaron de lado las consecuencias graves que se presentan ante una carencia de las moléculas combustibles. No se encontraron garantías ni apoyos. Por lo tanto, se catalogaron como afirmaciones sobre el tema sin justificación, lo que correspondió a un nivel de argumentación 0 .

Así mismo, se encontraron deficiencias en el concepto de las proteínas y en la diferencia entre tejido adiposo y muscular. Estos resultados indican que los conocimientos científicos relacionados con la química fueron adquiridos de forma general, lo que se debe posiblemente a la falta de complementariedad entre las asignaturas disciplinares y pedagógicas. Trabajos como los de Sadler y Fowler (2006) muestran que el conocimiento relacionado con las CSC influye significativamente en la práctica de la argumentación.

\section{Aportes de la argumentación a la formación de PQFI}

En las discusiones relacionadas con la toma de posición, los PQFI argumentaron sobre aspectos como: la influencia del medio ambiente sobre el enfermo, las consecuencias de no diagnosticar bien la enfermedad - como los tratamientos temporales y poco efectivos- y los costos que representa para el sistema de salud este tipo de patologías.

De otro lado, los PQFI identificaron como actores sociales al afectado, a la familia y a las instituciones médicas. También identificaron las causas que socialmente determinan la aparición de la enfermedad, como la publicidad, la presión social y los factores sicológicos.

Por eso, es importante que problemas sociocientíficos aparezcan en el currículo de ciencias, para no dejar en los estudiantes la impresión de que la ciencia no tiene ninguna relación con la vida cotidiana, ni plantea cuestiones éticas y sociales (Ratcliffe, 2009).

Según Zohar (2007), es importante que los profesores hayan adquirido conocimientos suficientes durante su formación que les permitan llevar procesos de argumentación en su aula de clase. Esto le facilitará al estudiante justificar sus opiniones, refutar argumentos y explicar por qué las opiniones de otros están equivocadas.

Teniendo en cuenta lo anterior, mediante los niveles de argumentación, se determinaron los conocimientos bioquímicos obtenidos por los PQFI en materias relacionadas como la química y cómo estos aportaron en su formación. Para ello, se realizó una entrevista en la que se trataron aspectos relacionados con la secuencia de enseñanza Discusiones argumentadas sobre el metabolismo de aminoácidos, en cuanto a la pertinencia de desarrollar los conceptos científicos del metabolismo de estas biomoléculas y de obtener, de esta manera, los argumentos necesarios para participar y realizar discusiones sobre el tema. 
Se encontró que los PQFI concuerdan en que la secuencia presentó una metodología novedosa que relaciona los temas de la bioquímica con una enfermedad que involucra varios aspectos - sociales, sicológicos, sociológicos, médicos, nutricionales y siquiátricos-. Por esa razón, se genera motivación para el aprendizaje a largo plazo de los conocimientos científicos de la disciplina.

Además, sostienen en sus intervenciones que, si esa herramienta de enseñanza aprendizaje se practica durante toda la carrera y desde el principio, muy probablemente sus aportes en los debates tendrían más elementos de juicio y de carácter científico. Les llama la atención el hecho de salirse de lo tradicional - texto, tablero, talleres, etc. - y de innovar creativamente con otros métodos de aproximación a los estudiantes. Sostienen, además, la importancia de una fundamentación científica en la disciplina para evitar que los juicios que se emiten en la argumentación se queden en niveles primarios que impidan la formulación de conclusiones suficientemente convincentes y apropiadas sobre los temas que se están tratando.

De igual forma, se hace especial énfasis en lo interesante que hubiera sido plantear un debate, el cual, por un lado, relacionara los resultados obtenidos en el laboratorio y la prevalencia de la enfermedad y, por otro, incluyera los síntomas, causas y consecuencias que se producen en el organismo cuando por la falta de alimentación se alteran los procesos químicos intracelulares, que involucran las moléculas que generan energía para los eventos metabólicos - biosíntesis de moléculas corporales, transporte activo de sustancias a través de la membrana, contracción del músculo, impulso nervioso, etc.-. De igual manera, hacen la observación con respecto a recordar las pautas para argumentar de forma científica como herramienta de la didáctica en el aprendizaje de los estudiantes.

De otro lado, la falta de profundidad de los conceptos que apoyan los temas tratados hace que el debate se presente en algunos momentos tedioso, repetitivo, superficial y centrado en el orden con el cual se gastan las moléculas que se incorporan en la dieta y las que se encuentran reservadas en el cuerpo, para hacer frente a un ayuno prolongado y voluntario, como en la anorexia.

Resaltan la importancia de abordar temas como el metabolismo de aminoácidos a través del planteamiento de situaciones específicas relacionadas con sucesos sociales. Así es más fácil aplicar los conceptos teóricos del syllabus. El debate académico motivado por un tema de actualidad y cercano permite un aprendizaje más duradero. Si no, por lo menos es posible que en el futuro - en el caso concreto de los aminoácidos- relacionen la información recibida por diferentes medios con las vías metabólicas, que se encargan de utilizar las moléculas que se reservan y las que se consumen para la obtención de energía útil.

Manifiestan también el valor de la actividad de laboratorio representada en la secuencia, la cual permite a los estudiantes demostrar experimentalmente los aspectos teóricos relevantes que se discuten en el aula, así como relacionar e interpretar los resultados y compararlos con los modelos diseñados para la comprensión en el caso concreto de las vías metabólicas. De otro lado, estas actividades facilitan procesos de indagación y de planteamiento de situaciones problémicas novedosas para la mejor comprensión del tema de la actividad experimental. Sin embargo, con la consulta previa a referentes bibliográficos de carácter científico y con el apoyo de las personas conocedoras del tema, es posible orientar en buena medida la deliberación previa a la experimentación para hacer una relación clara entre la teoría y la práctica. 
Los PQFI sugieren la necesidad de retroalimentar, de aclarar y de relacionar los resultados obtenidos con los aspectos metabólicos que se desencadenan con la anorexia.

Además, cuando se conoce el tema a profundidad, se puede organizar la secuencia en: la selección del tema, el contenido, las lecturas de apoyo, las preguntas orientadoras, el debate y las actividades experimentales. Otro factor para tener en cuenta cuando se trata de incorporar en el aula innovaciones didácticas es contar con el tiempo necesario que permita a los participantes consultar en diferentes referentes bibliográficos los conceptos por debatir. También es necesario contar con el apoyo de orientadores conocedores del tema que moderen la controversia y aporten elementos clave para llegar a conclusiones pertinentes.

\section{Conclusiones}

Teniendo en cuenta que el objetivo principal era analizar los procesos de argumentación desarrollados en PQFI a partir de la secuencia de enseñanza, se encontró que, aunque fue una herramienta interesante - la cual podrían llevar al aula de clase para propiciar mayor interés por parte de los estudiantes-, hubo dificultades en consolidar altos niveles de argumentación, los cuales permanecieron en los niveles básicos 0 y 1 . Por esta razón, la argumentación debe incorporarse de forma permanente y transversal en los currículos de los futuros profesores de química, para que sean capaces de enseñar de una manera flexible y creativa, que a la vez facilite un cambio didáctico (Zohar, 2007).

La argumentación se situó mayoritariamente en el nivel 1, donde los argumentos están relacionados con el metabolismo de aminoácidos. Es decir, son proposiciones requeridas. Los apoyos se basaron en la hidrólisis de proteínas para obtener aminoácidos útiles en producción de energía en casos extremos de ayuno, como el realizado voluntariamente por los anoréxicos. Se presenta un lenguaje científico, sin llegar a tener un alto nivel de argumentación - lo que implicaría construcción de garantías, calificadores y atención a refutaciones y excepciones-.

De igual manera, se encontraron niveles 0 , donde los PQFI daban afirmaciones aisladas o tomadas al azar sobre el metabolismo energético en general, razón por la cual no se encontraron apoyos relacionados con el tema en discusión. Así mismo, se determinó que solo una minoría de PQFI se situó en nivel 2, donde existen proposiciones que, por su contenido científico, se encuentran implícitas en el dato (Solow, 2006). Algunos presentaron apoyos bioquímicos, lo cual les permitió llegar de manera parcial a la conclusión.

En cuanto a la toma de posición, con el uso de una CSC controvertida como la anorexia, los PQFI plantearon una discusión en la que establecieron la importancia de conocer aspectos como los que involucran una imagen social, el medio que se desenvuelve el enfermo, los costos que implica la rehabilitación de este tipo de pacientes y las políticas que debería adoptar el gobierno para estos casos. Esta situación contribuye a desarrollar el pensamiento crítico que les permita cuestionar un discurso o los intereses mismos de la clase, además del desarrollo de capacidades argumentativas necesarias en caso de que existan posturas controvertidas sobre un tema científico que implique diferentes intereses y valoraciones (Solbes, 2013).

Así mismo, se estableció la necesidad de hacer mayor énfasis en los contenidos 
programáticos de la bioquímica como el del metabolismo energético. A la vez, se podría utilizar la interdisciplinariedad para hacer debates con más elementos de juicio.

Entre otros aspectos que ayudaron a favorecer la argumentación en los PQFI, está el trabajo de laboratorio con muestras de fluidos - orina - en pacientes para contrastarlas con las de personas que no sufren esta clase de trastornos, así como las preguntas y los artículos propuestos en la secuencia. Esto fomentó en parte la necesidad de investigar sobre conceptos científicos necesarios para el desarrollo de las actividades propuestas en la secuencia didáctica.

Los resultados evidencian que el abordaje de CSC tiene importantes posibilidades y aportes para mejorar la formación de profesores de química, de tal forma que puedan mejorar los procesos de enseñanza de la química en virtud de los siguientes aspectos, tal como ha sido documentado por Martínez y Parga (2013):

- El abordaje de CSC en la formación del profesor posibilita una mejor comprensión de conceptos científicos si hay una contextualización social y una perspectivainterdisciplinar, dado que es necesario relacionar dichos conceptos con su importancia social, en este caso, asociada al estudio de las causas e implicaciones sociales de la anorexia.

- El desarrollo de secuencias de enseñanza estructuradas a partir de CSC permite motivar el estudio de conceptos científicos abstractos, tales como los tratados en clases de bioquímica, lo cual favorece un mejor interés hacia su estudio y una mejor comprensión de la aplicación social que tiene el conocimiento científico.

El desarrollo de discusiones y las actividades de laboratorio contextualizadas con asuntos de salud - tales como los tratados en el desorden alimenticio generado por la anorexiapueden favorecer mejores procesos argumentativos en la formación de los futuros profesores de química.

Es necesario incluir la argumentación en la formación en química para favorecer propuestas innovadoras en enseñanza de la química a partir del diseño y dela implementación de secuencias didácticas que favorezcan mejores aprendizajes.

\section{Referencias}

Álvarez, A. (2008). La etnografía como modelo de investigación en educación. Gazeta de Antropología, 1, 1-16.

Driver, R., Newton, P., \& Osborne, J. (1997). Establishing the norms of scientific argumentation in classrooms [Ponencia]. ESERA Conference, Roma, Italia.

Driver, R., Newton, P., \& Osborne, J. (2000). Establishing the norms of scientific argumentation in classrooms. Science Education, 84(3), 287-312.

Gallo, D. (2013). Argumentación en profesores de química en formación inicial a partir de una secuencia de enseñanza sobre metabolismo de aminoácidos [Tesis de Maestría, Universidad Pedagógica Nacional]. Repositorio Institucional UPN. http://repository. pedagogica.edu.co/handle/20.500.12209/290

Martínez, L. (2012). Questões sociocientíficas na prática docente: ideologia, autonomia e formação de professores. Editora Unesp. 
Martínez, L. (2014). Cuestiones sociocientíficas en la formación de profesores de ciencias: aportes y desafíos. Tecné, Episteme y Didaxis, 36, 77-94.

Martínez, L., \& Parga, D. (2013). Discurso ético y ambiental sobre cuestiones sociocientíficas: aportes a la formación del profesorado de ciencias. UPN.

Marafioti, R. (2006). Argumentación. Análisis, evaluación, presentación. Biblos.

Moreira, M. (2002). Investigación en educación en ciencias: métodos cualitativos. Universidad de Burgos, Departamento de Didácticas Específicas.

Moreno, D., \& Martínez, L. (2009). Argumentación en estudiantes de educación media y habilidad del profesor para su desarrollo: una discusión en el aula sobre implicaciones sociales y ambientales de la producción de etanol. Nodos y Nudos, 3(27), 30-42.

Murillo, J., \& Martínez, C. (2010). Investigación etnográfica. http://www.rinace.net/javier. murillo/InvestigacionEE/Presentaciones/Curso_10/I_Etnografica_Trabajo.pdf

Liakopoulos, M. (2002). Análise argumentativa. En M. Bauer, \& G. Gaskell (Orgs.), Pesquisa qualitativa com texto, imagem e som. Um manual prático (2a ed., pp. 218-243). Vozes.

Leach, J., \& Scott, P. (2002). Designing and evaluating science teaching sequences: an approach drawing upon the concept of learning demand and a social constructivist perspective on learning. Studies in Science Education, 38, 115-142.

Ratcliffe, M. (2009). The place of socio-scientific issues in citizenship education. En A. Ross (Ed.), Human Rights and Citizenship Education (pp. 12-16). CiCe.

Sadler, T., \& Fowler, S. (2006). A threshold model of content knowledge transfer for socioscientific argumentation. Science Education, 90(6), 986-1004.

Sangiogo, F., Woyciechoswsky, R., \& Albrecht, S. (2011). A pesquisa educacional como atividade curricular na formação de licenciandos de química. Ciência \& Educação, 17(3), 523-540.

Solow, D. (2006). Introducción al razonamiento matemático. Limusa.

Solbes, J. (2013). Contribución de las cuestiones sociocientíficas al desarrollo del pensamiento crítico (I): introducción. Revista Eureka sobre Enseñanza y Divulgación de las Ciencias, 10(1), 1-10.

Suárez, M. (23 de junio de 2009). Las voces de la anorexia. El Espectador. https://www. elespectador.com/noticias/actualidad/las-voces-de-la-anorexia/

Toulmin, S. (2007). Los usos de la argumentación. Península.

Zenteno, M., \& Garritz, A. (2010). Secuencias dialógicas, la dimensión CTS y asuntos sociocientíficos en la enseñanza de la química. Revista Eureka sobre Enseñanza y Divulgación de las Ciencias, 7(1), 2-25.

Zohar, A. (2007). Science Teacher Education and Professional Development in Argumentation. En S. Erduran \& M. Jiménez (Eds.), Argumentation in Science Education (pp. 245-266). Springer.

Zubieta, L. (1982). Etnografía y política educativa. Revista Colombiana de Educación, 10, 107-115. 


\title{
ANEXO 1 \\ Cuestionario aplicado a los participantes de la investigación
}

\author{
(F) Universidad PEDAGOGICA \\ NACIONAL \\ NAdusadorade Educadores \\ FACULTAD DE CIENCIA Y TECNOLOGÍA \\ DEPARTAMENTO DE QUIIMICA \\ CUESTIONARIO DIRIGIDO A PROFESORES DE QUÍMICA EN \\ FORMACIÓN INICIAL
}

Este cuestionario electrónico pretende recoger algunas informaciones sobre su formación universitaria, importante para el trabajo de investigación sobre argumentación en cuestiones sociocientíficas de los profesores en formación. Agradecemos su diligenciamiento

\section{Fecha:}

Nombre y apellidos:

Sexo: $F \quad M$

Edad:

Semestre:

1. De acuerdo con el siguiente cuadro marque con una $\mathrm{X}$ debajo de cada materia, las que ha aprobado

\begin{tabular}{|l|l|l|l|l|l|}
\hline $\begin{array}{l}\text { Teorías } \\
\text { Químicas I }\end{array}$ & $\begin{array}{l}\text { Formación } \\
\text { Matemática } \\
\text { I }\end{array}$ & $\begin{array}{l}\text { Teorias } \\
\text { Físicas } \\
\text { I }\end{array}$ & $\begin{array}{l}\text { Educación } \\
\text { y Sociedad }\end{array}$ & $\begin{array}{l}\text { Pedagogía y } \\
\text { Didáctica I }\end{array}$ & $\begin{array}{l}\text { Formación } \\
\text { Comunicativa } \\
\text { (ingles I) }\end{array}$ \\
\hline $\begin{array}{l}\text { Teorías } \\
\text { Químicas II }\end{array}$ & $\begin{array}{l}\text { Formación } \\
\text { Matemática }\end{array}$ & $\begin{array}{l}\text { Teorías } \\
\text { Físicas } \\
\text { II }\end{array}$ & $\begin{array}{l}\text { Psicología } \\
\text { Cognitiva }\end{array}$ & $\begin{array}{l}\text { Pedagogía y } \\
\text { Didáctica II }\end{array}$ & $\begin{array}{l}\text { Formación } \\
\text { Comunicativa } \\
\text { (ingles II) }\end{array}$ \\
\hline $\begin{array}{l}\text { Teorías } \\
\text { Químicas III }\end{array}$ & $\begin{array}{l}\text { Formación } \\
\text { Matemática }\end{array}$ & $\begin{array}{l}\text { Teorias } \\
\text { Físicas } \\
\text { III }\end{array}$ & $\begin{array}{l}\text { Teorías } \\
\text { Curriculares }\end{array}$ & $\begin{array}{l}\text { Pedagogía y } \\
\text { Didáctica III }\end{array}$ & $\begin{array}{l}\text { Informática } \\
\text { Educativa I }\end{array}$ \\
\hline $\begin{array}{l}\text { Sistemas } \\
\text { Orgánicos I }\end{array}$ & $\begin{array}{l}\text { Sistema } \\
\text { Inorgánicos } \\
\text { I }\end{array}$ & $\begin{array}{l}\text { Sistemas } \\
\text { Biológicos }\end{array}$ & $\begin{array}{l}\text { Historia y } \\
\text { Epistemología } \\
\text { De la Química }\end{array}$ & $\begin{array}{l}\text { Formación } \\
\text { Comunicativa } \\
\text { (español) }\end{array}$ & $\begin{array}{l}\text { Informática } \\
\text { Educativa II }\end{array}$ \\
\hline
\end{tabular}




\begin{tabular}{|c|c|c|c|}
\hline $\begin{array}{l}\text { Sistemas } \\
\text { Orgánicos ॥ }\end{array}$ & $\begin{array}{l}\text { Sistema } \\
\text { Inorgánicos } \\
\text { II }\end{array}$ & \begin{tabular}{|l|} 
Legislación \\
Educativa
\end{tabular} & $\begin{array}{l}\text { Formación } \\
\text { Filosófica }\end{array}$ \\
\hline $\begin{array}{l}\text { Métodos de } \\
\text { Análisis } \\
\text { Químico I }\end{array}$ & $\begin{array}{l}\text { Sistemas } \\
\text { Fisicoquímicos } \\
\text { I }\end{array}$ & $\begin{array}{l}\text { Formulación y } \\
\text { Gestión de } \\
\text { Proyectos } \\
\text { Educativos }\end{array}$ & $\begin{array}{l}\text { Educación y } \\
\text { Participación } \\
\text { Ciudadana }\end{array}$ \\
\hline $\begin{array}{l}\text { Métodos de } \\
\text { Análisis } \\
\text { Químico II }\end{array}$ & $\begin{array}{l}\text { Sistemas } \\
\text { Fisicoquímicos } \\
\text { II }\end{array}$ & $\begin{array}{l}\text { Práctica } \\
\text { Pedagógica y } \\
\text { Didáctica I }\end{array}$ & $\begin{array}{l}\text { Educación } \\
\text { ambiental }\end{array}$ \\
\hline $\begin{array}{l}\text { Sistema } \\
\text { Bioquímicos }\end{array}$ & \begin{tabular}{|l|} 
Sistemas \\
Biológicos II
\end{tabular} & $\begin{array}{l}\text { Práctica } \\
\text { Pedagógica y } \\
\text { Didáctica II }\end{array}$ & \\
\hline
\end{tabular}

2. ¿En alguna de las materias cursadas durante su carrera estudio algún aspecto relacionado con argumentación? Si No

2.1.1. Si respondió afirmativamente el anterior ítem ¿En cuál o cuáles materias estudio sobre argumentación?

2.1.2. Describa los aspectos que fueron estudiados en la(s) anterior(es) materia(s) relacionados con el tema

3. ¿En alguna de las materias cursadas durante su carrera estudio algún aspecto relacionado con cuestiones sociocientíficas? $\mathrm{Si} \_$No

3.1. Si respondió afirmativamente el anterior ítem ¿En cuál o cuáles materias estudio sobre argumentación?

3.2. Describa el (los) aspecto(s) que estudio durante su pregrado relacionados con cuestiones sociocientíficas.

4. ¿Durante su práctica docente desarrolló alguna actividad relacionada con argumentación o en cuestiones socientificas? Si _ No_ 
4.3.1. Si respondió afirmativamente el anterior ítem describa qué actividad docente desarrollo relacionando estos temas.

5. Con los conocimientos adquiridos sobre aminoácidos conteste los ítems propuestos

Un paciente refiere al médico los siguientes síntomas: falta de sueño, temperatura baja, falta de apetito, mal genio. En el diagnostico expedido se encuentra que una de las causas son los niveles bajos de serotonina.

La reacción general para la obtención de este neurotransmisor es

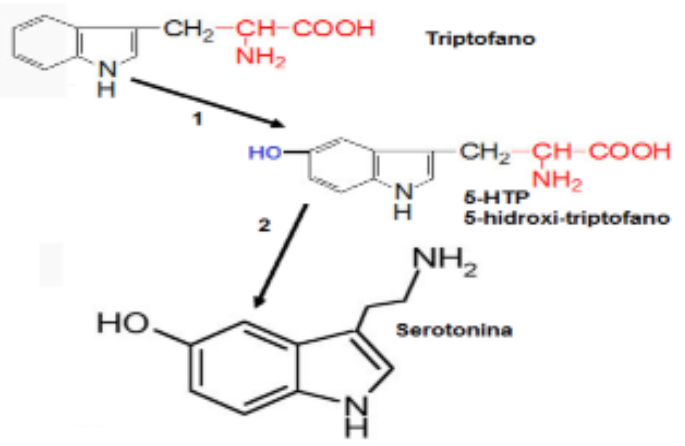

- Con la estructura del triptófano explique químicamente que forma iónica tiene a pH fisiológico

- Observe las estructuras de la 5-HTP y la serotonina y comente de forma breve los cambios que realizan las enzimas sobre el triptófano.

- Según el diagnóstico realizado por el médico, este aminoácido es indispensable para el ser humano. ¿Usted apoya o refuta esta información? Argumente de forma breve su respuesta.

6. Reflexionando sobre una cuestión social

"la muerte por hambre de una niña, a principios de marzo, paso inadvertida. Se supo que murió a los cuatro años, que tenía un nombre bastante sonoro, Janelda, que era chocoana. El diagnóstico: desnutrición crónica severa".

Tomado del Colombiano 23 de junio 2012

Argumente brevemente sobre la importancia de los aminoácidos en la nutrición. Tenga en cuenta estructura química, reacciones de estas biomoléculas. 